\title{
La transformation numérique, ou la métamorphose d'une chenille en papillon
}

Écrit par : Angel Gurría, Secrétaire général de l’OCDE

Dernière mise à jour : 7 juin 2019

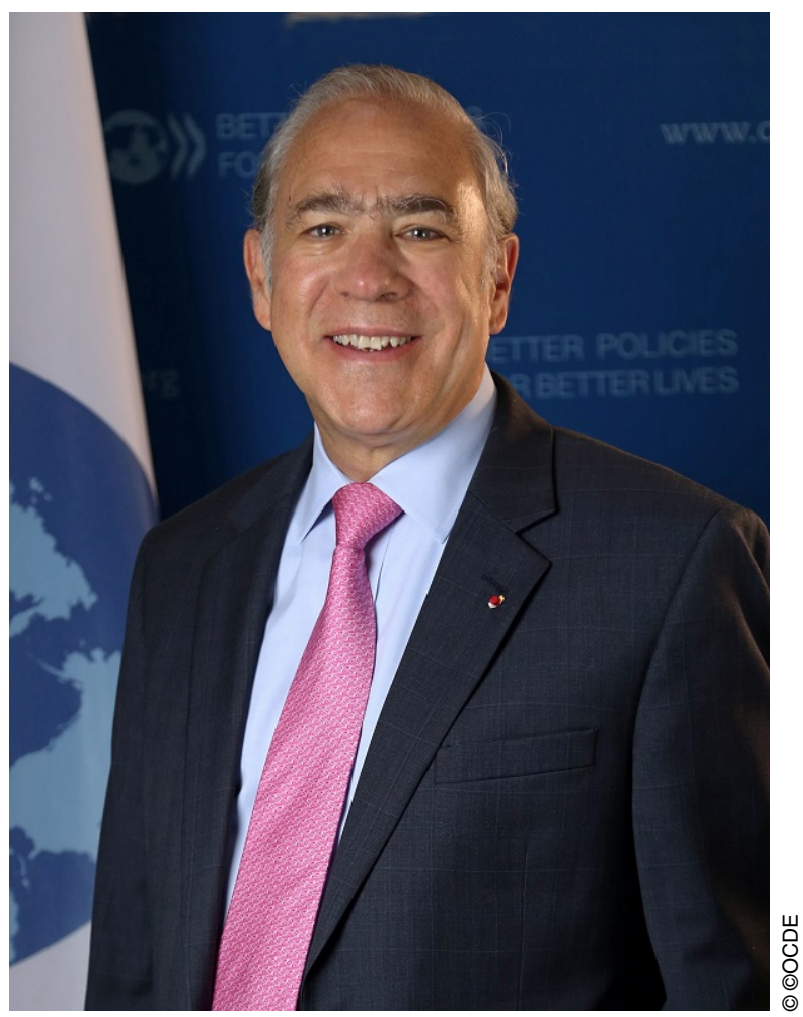

La transformation numérique n'est certes pas nouvelle, mais le rythme des évolutions s'accélère et nos sociétés ultraconnectées génèrent des volumes considérables de données de toutes sortes.

Ces flots de données transforment la création de valeur, les processus décisionnels et la production, tout comme l'invention de nouveaux matériaux ou des moteurs à combustion a jadis révolutionné les économies et les sociétés.

La transformation à l'œuvre offre d'innombrables possibilités d'améliorer le bienêtre, dans des domaines aussi variés que les soins de santé, l'éducation et l'environnement. Le smartphone peut ainsi utiliser l'IA afin de détecter d'éventuels problèmes de santé, ou la reconnaissance vocale pour proposer une 
traduction à la volée. Des robots équipés de caméras peuvent inspecter l'intérieur des oléoducs à la recherche de fissures et empêcher par là même des dégâts environnementaux.

Pourtant, ces avantages s'accompagnent de nouvelles difficultés. La transformation numérique suscite des inquiétudes quant à la polarisation des emplois et l'inadéquation des compétences, aux violations de la vie privée et de la sécurité, au pouvoir de marché grandissant des entreprises de pointe, ou encore à la fraude et la planification fiscale agressive. Elle pose également des problèmes d'inclusion et d'égalité : plus de 4 ménages ruraux sur 10 n'ont pas accès à une connexion à très haut débit et seulement $11 \%$ des entreprises effectuent des analyses des données massives.

Les effets positifs et négatifs de la transformation mettent les décideurs au défi d'évaluer les coûts et les avantages des différentes possibilités d'action publique. Interdire le covoiturage parce que cela contrevient aux règles d'exercice de la profession de conducteur de taxi peut en effet compliquer la tâche des travailleurs pauvres qui y ont recours pour se rendre sur leur lieu de travail. De même, une limitation excessive de l'accès aux données au titre de la confidentialité peut priver la recherche sur la démence de précieuses informations. D'un autre côté, un trop grand laxisme peut favoriser une concentration du pouvoir, voire ébranler les institutions démocratiques.

Si, sur le plan national, ces problématiques n'épargnent quasiment aucun aspect des politiques, elles dépassent également le cadre des frontières, notamment pour ce qui est des échanges numériques. À tel point qu'elles figurent désormais en bonne place parmi les priorités des institutions de l'ONU - dont l'UIT et l'UNESCO -, ainsi que d'autres instances internationales, telles le G20, le G7, l'UE, l'APEC, sans oublier l'OCDE.

En 2016, les ministres réunis à Cancún ont reconnu la nécessité d'une approche cohérente multipartite de la transformation numérique et lancé le projet de l'OCDE «Vers le numérique ». Les travaux, qui ont débuté en 2017, mobilisent 10 Directions de l'OCDE et plus de 14 comités d'orientation.

Dès l'origine, le but était de parvenir à des solutions centrées sur l'humain, en faisant des technologies numériques un vecteur d'autonomisation et non pas d'asservissement ou d'appauvrissement.

Cet effort, nourri par les meilleures évaluations, analyses et réflexions stratégiques, a abouti à l'élaboration d'un outil unique en son genre à l'intention des gouvernements : le Cadre d'action intégré du projet de l'OCDE « Vers le numérique ».

C'est la première fois qu'une approche globale de la transformation numérique est formulée. [...] 
Nous voulons réduire les fractures numériques entre les individus et entre les entreprises. Nous voulons donner aux individus les moyens de réussir dans un monde du travail irrigué par le numérique, en rapide évolution. Nous voulons renforcer la confiance et élargir l'accès aux données pour stimuler l'innovation. Enfin, nous voulons bâtir la nouvelle génération de données et d'indicateurs qui nous aideront à surveiller et façonner la transformation numérique. Il s'agit là d'un projet certes ambitieux, mais, avec votre concours, l'OCDE peut faire des miracles.

Pour réussir la transformation numérique, il convient de préparer les individus et les pouvoirs publics à mettre les technologies numériques au service du développement humain. George Westerman, chercheur au MIT, l'affirme : « Si elle est bien réalisée, la transformation numérique s'apparente à la métamorphose d'une chenille en papillon. Dans le cas contraire, vous vous retrouvez juste avec une chenille très rapide ". L'OCDE est prête à vous accompagner dans votre propre métamorphose.

Pour en savoir plus sur le projet «Vers le numérique », rendez-vous sur : $\underline{\text { https:// }}$ www.oecd.org/going-digital/

D’après le discours liminaire du Secrétaire général lors du Sommet de l'OCDE sur la transformation numérique : Les promesses de la transformation numérique, le 11 mars 2019. La version originale complète est consultable à l'adresse : $\underline{\text { https:// }}$ oe.cd/2wJ 